\title{
Feedback Survey of the Effect, Burden, and Cost of the National Endoscopic Quality Assessment Program during the Past 5 Years in Korea
}

\author{
Yu Kyung Cho ${ }^{1}$, Jeong Seop Moon ${ }^{2}$, Dong Su Han ${ }^{3}$, Yong Chan Lee ${ }^{4}$, Yeol Kim ${ }^{5}$, Bo Young Park ${ }^{5}$, II-Kwun Chung ${ }^{6}$, Jin-Oh Kim ${ }^{7}$, \\ Jong Pil $\mathrm{Im}^{8}$, Jae Myung Cha ${ }^{9}$, Hyun Gun Kim${ }^{7}$, Sang Kil Lee ${ }^{4}$, Hang Lak Lee ${ }^{3}$, Jae Young Jang ${ }^{9}$, Eun Sun Kim ${ }^{10}$, Yunho Jung ${ }^{6}$, \\ Chang Mo Moon ${ }^{11}$ and Ethics and Quality Control Committee of Korean Society of Gastrointestinal Endoscopy
}

${ }^{1}$ Department of Internal Medicine, College of Medicine, The Catholic University of Korea, Seoul, ${ }^{2}$ Department of Internal Medicine, Inje University Seoul Paik Hospital, Inje University College of Medicine, Seoul, ${ }^{3}$ Department of Internal Medicine, Hanyang University Guri Hospital, Hanyang University College of Medicine, Guri, ${ }^{4}$ Department of Internal Medicine, Yonsei University College of Medicine, Seoul, ${ }^{5}$ Cancer Early Detection Branch, National Cancer Control Institute, National Cancer Center, Goyang, ${ }^{6}$ Department of Internal Medicine, Soonchunhyang University Cheonan Hospital, Soonchunhyang University College of Medicine, Cheonan, ${ }^{7}$ Department of Internal Medicine, Soonchunhyang University College of Medicine, Seoul, ${ }^{8}$ Department of Internal Medicine, Seoul National University College of Medicine and Liver Research Institute, Seoul, ${ }^{9}$ Department of Internal Medicine, Kyung Hee University School of Medicine, Seoul, ${ }^{10}$ Department of Internal Medicine, Korea University College of Medicine, Seoul, ${ }^{11}$ Department of Internal Medicine, Ewha Womans University School of Medicine, Seoul, Korea

Background/Aims: In Korea, the nationwide gastric cancer screening program recommends biennial screening for individuals aged 40 years or older by way of either an upper gastrointestinal series or endoscopy. The national endoscopic quality assessment (QA) program began recommending endoscopy in medical institutions in 2009. We aimed to assess the effect, burden, and cost of the QA program from the viewpoint of medical institutions.

Methods: We surveyed the staff of institutional endoscopic units via e-mail.

Results: Staff members from 67 institutions replied. Most doctors were endoscopic specialists. They responded as to whether the QA program raised awareness for endoscopic quality $(93 \%)$ or improved endoscopic practice $(40 \%)$. The percentages of responders who reported improvements in the diagnosis of gastric cancer, the qualifications of endoscopists, the quality of facilities and equipment, endoscopic procedure, and endoscopic reprocessing were $69 \%, 60 \%, 66 \%, 82 \%$, and $75 \%$, respectively. Regarding reprocessing, many staff members reported that they had bought new automated endoscopic preprocessors (3\%), used more disinfectants (34\%), washed endoscopes longer (28\%), reduced the number of endoscopies performed to adhere to reprocessing guidelines (9\%), and created their own quality education programs (59\%). Many responders said they felt that QA was associated with some degree of burden (48\%), especially financial burden caused by purchasing new equipment. Reasonable quality standards (45\%) and incentives (38\%) were considered important to the success of the QA program.

Conclusions: Endoscopic quality has improved after 5 years of the mandatory endoscopic QA program.

Clin Endosc 2016;49:542-547

Key Words: Endoscopy; Quality; Mass screening; Stomach neoplasms

Received: September 8, 2015 Revised: October 6, 2015

Accepted: October 6, 2015

Correspondence: Jeong Seop Moon

Department of Internal Medicine, Inje University Seoul Paik Hospital, Inje University College of Medicine, 9 Mareunnae-ro, Jung-gu, Seoul 04551, Korea

Tel: +82-2-2270-0012, Fax: +82-2-2279-4021, E-mail: moonjs2@unitel.co.kr

(c) This is an Open Access article distributed under the terms of the Creative Commons Attribution Non-Commercial License (http://creativecommons.org/ licenses/by-nc/3.0) which permits unrestricted non-commercial use, distribution, and reproduction in any medium, provided the original work is properly cited.

\section{INTRODUCTION}

In Korea, a nationwide gastric cancer screening program was started in 1999 as part of the National Cancer Screening Program (NCSP). ${ }^{1}$ The NCSP recommends biennial gastric cancer screening for men and women aged 40 years or older by way of either an upper gastrointestinal series or endoscopy. ${ }^{2}$ The gastric cancer screening program has reduced the 
incidence and mortality of gastric cancer, but the degree of success is highly variable. ${ }^{3}$

The National Endoscopy Quality Assessment program was initiated in 2009 for quality control of endoscopic screening protocols. The National Cancer Center and the National Health Insurance Service (NHIS) organized the program in cooperation with the Korean Society of Gastrointestinal Endoscopy (KSGE). ${ }^{4,5}$ For medical institutions that perform screening endoscopies, five domains of endoscopic quality are assessed annually: qualifications of endoscopists, endoscopic unit facility and equipment, endoscopic procedure, outcome of endoscopy, and endoscopic reprocessing.

To achieve successful implementation of the quality assessment (QA) program, good communication between the testing institution and the tested medical institution is essential. We surveyed medical institutions nationwide on their perceptions of the endoscopic QA program. We aimed to identify the influences on real practice, key components for success of the QA program, and level of difficulty of implementation from the viewpoint of each institution.

\section{MATERIALS AND METHODS}

This survey was conducted from November 2014 to January 2015 by the KSGE ethics and quality control committee. An e-mail-based or post-based survey was utilized. The KSGE ethics and quality control committee approved the questionnaire. More than a hundred doctors or head nurses working in medical institutions or health-screening centers that perform screening endoscopy were invited to participate. The anonymous responses were collected after 1 week either by post or in person by committee members.

\section{Survey development and structure}

A comprehensive questionnaire was developed through review of the current endoscopic QA protocol and literature. The questionnaire dealt with self-assessment of endoscopic quality in all measurable domains, the costs associated with the program, and the administrative burden felt during preparation of the QA audit. All responses were anonymous, and the questionnaire was designed to take 15 to 20 minutes to complete. It consisted of several sections: (1) responder demographics; (2) questions regarding current status of the national endoscopic QA program; (3) questions regarding the proper management of institutions that did not pass the audit; (4) questions regarding how much the quality has improved (self-assessment); (5) questions regarding burden and cost; and (6) quality improvement (QI) of endoscopic reprocessing.

\section{RESULTS}

\section{General characteristics of the endoscopic units}

Doctors and nurses in the endoscopic units of 67 medical institutions responded to the survey. Among them, 79\% were nurses, and the remainder were doctors. The median numbers of doctors and nurses from each institution were 14 (interquartile range [IQR], 5 to 15 ) and 14 (IQR, 8 to 16 ). More than one endoscopic specialist worked at 54 institutions, all doctors were endoscopic specialists at 39 institutions, and doctors worked as endoscopists at 13 institutions. The average numbers of endoscopies and colonoscopies performed per year were more than 3,000 at 50 institutions and more than 100 at 42 institutions. Thirty-three institutions had large endoscopic units performing more than 10,000 endoscopic screenings per year (Table 1).

\section{Questions regarding the current status of the na- tional endoscopic QA audit}

Regarding the question, "How do you perceive the effect of the QA program?" $93 \%$ of responders said that the national QA program raised awareness of endoscopic quality and improved their practices $(n=27,40 \%)$, whereas others answered that their practices had not changed much $(n=40,53 \%)$. The remainder answered that their perceptions of endoscopic quality had not changed despite the QA program $(n=3,6 \%)$

Table 1. General Subject Characteristics

\begin{tabular}{lc}
\hline Characteristic & Value \\
\hline No. of medical institutions involved in this survey & 67 \\
\hline No. of doctors, median (IQR) & $14(5-15)$ \\
\hline No. of nurses, median (IQR) & $14(8-16)$ \\
\hline No. of nursing assistants, median (IQR) & $3(1-3)$ \\
\hline No. of other members, median (IQR) & $1(1-2)$ \\
\hline Specialties of the doctors & \\
\hline Physician+Endoscopic specialist \pm Family doctor & 14 \\
\hline Physicians & 13 \\
\hline General surgeon+Endoscopic specialist & 1 \\
\hline Endoscopic specialist & 39 \\
\hline Mean no. of endoscopies performed/year & \\
\hline 10,000 or more & 33 \\
\hline $5,000-9,999$ & 12 \\
\hline $3,000-4,999$ & 5 \\
\hline $1,000-2,999$ & 13 \\
$500-999$ & 2 \\
\hline 500 & 2 \\
\hline
\end{tabular}

IQR, interquartile range. 
Table 2. Questions Regarding the Current Status of the Endoscopic Quality Assessment Program

How much did you feel the endoscopic quality assessment (QA) program influenced endoscopic practices in the institution?

Successful (It improved endoscopic practices)

Somewhat successful (It raised awareness of endoscopic quality, but practice has not changed much)

The perception of endoscopic quality has not changed.

If you feel that the endoscopic quality improvement (QI) program has not been successful, what do you think the causes are? (Double choices permitted)

The standards ignore the realities of medical institutions

Too much administrative burden to prepare it

Cost to prepare it

Indifference to it

Psychological aversion to it

6

Inability to comprehend the standards

What do you think is the most important factor needed to make the endoscopic QI program successful? (Double choices permitted)

The standard considering the reality of the practices

45

Incentives (all types) for institutions that achieve QA in practice

38

The change in perception of the quality of medical institutions

Effective promotions for the QA program

25

Cooperation from medical institutions

Table 3. Questions Given to Those Who Did Not Pass the Endoscopic Quality Improvement Program

\begin{tabular}{l|c}
\hline & Number \\
\hline If you were chosen for reeducation, what do you think the reason was? & 30 \\
\hline Our unit has a high endoscopic volume. & 11 \\
\hline We did not prepare the quality improvement (QI) audit properly. & 2 \\
\hline Our endoscopic unit facility is poor. & 1 \\
\hline The diagnostic sensitivity for cancer is low. & 0 \\
\hline We have poor endoscopic reprocessing. & 25 \\
\hline We would not be chosen to be reeducated (or question not answered). & 50 \\
\hline What do you think would be the proper management strategy for institutions that did not pass the audit? & 9 \\
\hline Reeducation & 8 \\
\hline Repeat of audit until they pass & 34 \\
\hline Warning or penalty & 16 \\
\hline Do you disagree with warnings or penalties? If so, why? & 11 \\
\hline Disagree & 7 \\
\hline Penalties are not good methods for achieving quality assessment (QA). \\
\hline Accurate audits are difficult under the current criteria. \\
\hline The penalties imposed on medical institutions are too harsh.
\end{tabular}

\section{(Table 2).}

Regarding the question, "What do you think is the most important factor for the success of the endoscopic QA program?" most responders chose acceptable quality standards for the medical institution $(n=45)$. Other answers included incentives (all types) for institutions that achieved high-quality practice $(n=28)$ and changes in institutions' perceptions. Regarding the question, "If you feel that the QA program has 


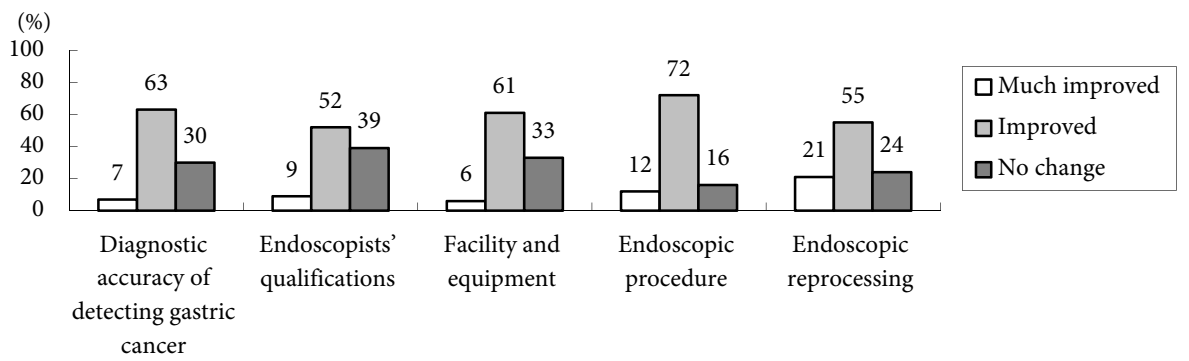

Fig. 1. Improvements in endoscopic quality after 5 years of the endoscopic quality improvement program. The data are shown as percentages.

not been successful, what do you think the causes are?" most responders said that standards that ignore the needs of real practice $(n=29)$ or too much of an administrative $(n=28)$ or financial $(n=25)$ burden.

\section{Regarding the question "what is the proper manage- ment strategy for institutions that did not pass the audit?"}

Regarding the question, "If you have to be reeducated, why do you think this is the case?" more than $50 \%$ of responders said they were chosen because their units are high-volume endoscopic centers rather than because of a low quality score. Others said it was because they had not prepared the audit well, or they had a poor unit facility, or they had low diagnostic accuracy for gastric cancer. There was no answer regarding poor endoscopic processing. Regarding the proper management strategy for institutions that did not pass the audit, 50\% of participants identified reeducation as the proper protocol (Table 3).

\section{Questions regarding how much the quality has im- proved}

When asked to self-assess their degree of improvement, $60 \%, 66 \%, 80 \%$, and $82 \%$ of participants reported improvements in diagnostic accuracy for detecting gastric cancer, improvements in qualifications of endoscopists (which involves more continuing medical education [CME] courses on gastrointestinal endoscopy), improvements in management of endoscopic unit facilities, and improvements in endoscopic procedure, respectively. Regarding endoscopic reprocessing, $75 \%$ responded that it had improved, and $21 \%$ responded that it had greatly improved (Fig. 1).

\section{Questions regarding burden and cost}

Of the responders, $52 \%$ were conscious of considerable burden and/or high costs while they prepared the endoscopic QA audit (Table 4). Regarding the question, "What were the difficulties encountered while preparing the QA audit?" most responders answered that they could not be sure whether they had prepared well $(n=29)$, or that facility standards were
Table 4. What Were the Costs and Administrative Burdens of Preparing the Endoscopic Quality Assessment Audit?

\begin{tabular}{lr}
\hline & No. (\%) \\
\hline Negligible, but the practice got somewhat disrupted & $8(12)$ \\
Not too much & $24(36)$ \\
Considerable burden or cost & $35(52)$ \\
\hline
\end{tabular}

not clearly defined $(n=21)$, or that they had had difficulty in meeting the criteria $(n=21)$. Proving total endoscopic volume $(n=6)$ or obtaining CME $(n=3)$ in regard to endoscopists' qualifications was difficult. The other answers regarded how they check frequently asked questions, how they downloaded documents or forms, how they were notified of audit results, and how they perceived the results.

Regarding costs spent in preparing the QA audit, 54\% of participants answered that they had attended CME courses more than twice to meet the criteria for qualification; $7 \%$ and $3 \%$ had bought new endoscopes or colonoscopes, respectively, to meet facility criteria; and $3 \%$ had purchased endoscopic hemostatic devices (e.g., hemoclips), emergency supplies (emergency drugs such as epinephrine, Ambu bags, laryngoscopes), emergency carts, or pulse oximeters. Some staff members said they had bought new containers for endoscopic storage (18\%), new automated endoscopic preprocessors (3\%), and supplies for endoscopic reprocessing (4\%). Regarding administrative costs, some responders referenced new documents for endoscopic procedures, new endoscopic reports (23\%), new informed consent protocols for sedated endoscopy (24\%), new discharge criteria (16\%), drug treatment guidelines (19\%), and pre-endoscopy checklists (24\%).

\section{Quality improvement of endoscopic reprocessing}

Regarding endoscopic reprocessing, $21 \%$ of staff members answered that it had been improved significantly. Some responders said they had bought a new automated endoscopic preprocessor (3\%), and some reported greater consumption of disinfectants (34\%), longer washing times $(28 \%)$, or reduced numbers of endoscopies per day to adhere strictly to reprocessing guidelines (9\%). Others reported they had bought new 
washing or reprocessing equipment (10\%). In addition, $60 \%$ of institutions had designed their own education programs to control endoscopic quality; $41 \%$ of participants said they already had their own autoclave or ethylene oxide gas sterilizer; $85 \%$ of staff said they had undergone education for endoscopic reprocessing; and $31 \%$ said they had designed their own reprocessing education protocols.

\section{DISCUSSION}

Although the incidence and mortality rates of gastric cancer are decreasing, it remains one of the major cancers in Korea. The NCSP, launched in 1999, provides free cancer screening for common cancers, including gastric cancer. The gastric cancer screening program recommends biennial gastric cancer screening for those aged 40 years or older by way of either an upper gastrointestinal series or endoscopy. Korea is the only country that uses endoscopy for gastric cancer screening. ${ }^{3}$

In Korea, increasing the quality of endoscopy has become an important priority in recent years. That agenda became organized into a more coordinated effort led by the National Cancer Center, the NHIS, and the KSGE. A national endoscopy QA program, initiated in 2009, aimed to enhance the quality of medical institutions and improve the effectiveness of the NCSP. Korean endoscopic quality standards were initially designed by a broad systemic review of other endoscopic quality guidelines and discussions with experts. ${ }^{6-8}$ The standards comprise five domains: qualifications of endoscopists, endoscopic unit facilities and equipment, endoscopic procedure, outcomes of endoscopy, and endoscopic reprocessing. Quality standards have improved in the past 5 years, supported by the KSGE and the NHIS. All institutions at which endoscopic screening is performed should undergo an annual QA audit implemented by the NHIS. We aimed to investigate the effects of the national endoscopic QA program on endoscopic practice and perceptions, burden, and costs of QA activityespecially from the viewpoints of medical institutions that conduct endoscopic screening.

The most-promising results of this survey are the perception that QI has enhanced significantly at $93 \%$ of the medical institutions since the national endoscopic QA program was initiated. QI even improved endoscopic practice in $40 \%$ of practices but had not yet influenced practice in 53\%. Those results show that the QA program is somewhat successful.

Approximately $80 \%$ of responders answered that all aspects of endoscopic quality had improved: qualifications of endoscopists, the endoscopic unit facility and equipment, endoscopic procedure and outcomes, and reprocessing. The rate of detection of gastric cancer had also increased, according to $80 \%$ of responders. CME in endoscopy for nonendoscopic specialists is mandatory to meet the criteria for endoscopists' qualifications, and $60 \%$ of participants answered that they had taken additional CME courses to prepare for the QA audit despite that the majority were already endoscopic specialists. Such education explains indirectly why detection of gastric cancer has increased since implementation of the QA program. ${ }^{9,10}$

QIs in the endoscopic unit facility and reprocessing were prominent. Quality indicators of endoscopic reprocessing include endoscope washing time, amounts of disinfectant used, and strict adherence to reprocessing guidelines, all of which were improved. Education and importance perception regarding reprocessing have been widespread. Some institutions said they had bought new automated preprocessors at high cost. Endoscopic reprocessing is an important issue associated with patient safety. The national QA program emphasizes strict adherence to endoscopic reprocessing guidelines. A qualitative evaluation by the KSGE in 2014 revealed several problems, such as reuse of disposable accessories, transport of contaminated endoscopes in a sealed container, and lack of eyewear, all of which require improvement. ${ }^{11}$ Most responders selected (1) financial problems such as medical insurance fees for endoscopic procedures and (2) large quantities of endoscopic examinations as the two most common causes of difficulty in complying with guidelines. However, the KSGE survey showed that the quality of endoscopic reprocessing has improved compared with results from the survey conducted 5 years previous. Periodic surveillance and practitioners' education in reprocessing should be continued.

Many participants indicated that adherence to quality standards should be rigorous, considering the current realities of medical institutions. Because screening endoscopy can be performed in small Korean clinics by nonendoscopic specialists to meet nationwide demand, the target level of the facility or equipment is likely to meet only minimum quality requirements. In particular, endoscopic facilities' quality standards were set based on small clinic size. Despite that, most responders complained that their facilities' ability to meet the quality criteria was one of the most difficult aspects of the program. In reality, anyone interested in the national QA program can easily access the protocols online and download the documents. Effective and intense promotions to the public represent an important strategy for overcoming indifference and psychological aversion.

Finding small defects early would reduce errors, lower overall costs, and improve patient satisfaction. ${ }^{12}$ But despite the advantages of the program, the preparation of a QA audit represents a financial and administrative burden from the viewpoint of medical institutions. In this survey, medical 
institutions said they had taken on the cost of endoscopists' $\mathrm{CME}$ and bought new equipment such as endoscopes or colonoscopes and even new automatic reprocessors. Creating new documents, implementing new systems, and designing education protocols for quality or reprocessing would be costly and time-consuming. The heavy workload and cost may justify criticisms that say the current standard does not consider the reality of clinics or institutions despite the fact that most of the institutions surveyed had large endoscopic units.

There are a few limitations to this study. The results of the survey depend largely on responders' characteristics. First, we did not select responders randomly, even though we had intended to choose responder candidates from high-volume endoscopy centers-ones performing more than 500 endoscopies per year. Practically, the majority of screening endoscopy is concentrated at the large medical institutions, and the national QA program prioritizes improvements to quality at those institutions. Second, responses that claimed QI may be overestimated because responders were biased in favor of large medical institutions or large health-screening centers.

This study is a subjective survey, with no objective QI data. It may not have measured endoscopic quality accurately. The ideal, objective parameter of QI in screening endoscopy would be the diagnostic accuracy of gastric cancer, but it is impossible to so measure that at one medical institution annually, considering the volume of endoscopy performed. In addition, there is no validated endoscopic quality indicator such as adenoma detection rate or cecal intubation rate in colonoscopy. Perceptional change is the important first step in taking action to improve quality. Therefore, the survey can be considered an indirect parameter measuring endoscopic quality from the point of view of participants in QI practice.

In conclusion, the quality of endoscopy has improved after 5 years of implementation of the mandatory endoscopic QA program. A continuous and well-organized national endo- scopic QI program is required for enhancing the effectiveness of national gastric cancer screening. The results of this survey provide a road map for developing a better quality assurance program for endoscopic practices.

\section{Conflicts of Interest}

The authors have no financial conflicts of interest.

\section{Acknowledgments}

This study was supported by National Cancer Center Grant (1560460-1).

\section{REFERENCES}

1. Shin A, Kim J, Park S. Gastric cancer epidemiology in Korea. J Gastric Cancer 2011:11:135-140.

2. Park HA, Nam SY, Lee SK, et al. The Korean guideline for gastric cancer screening. J Korean Med Assoc 2015;58:373-384.

3. Suh M, Choi KS, Lee YY, Park B, Jun JK. Cancer screening in Korea, 2012: results from the Korean National Cancer Screening Survey. Asian Pac J Cancer Prev 2013;14:6459-6463.

4. Chung IK. Quality assessment of gastroscopy in the national cancer screening. Korean J Gastrointest Endosc 2009;39(Suppl 1):53S-64S.

5. Han DS. The necessity and current state of endoscopy quality control. Korean J Gastrointest Endosc 2009;39(Suppl 1):50S-52S.

6. Armstrong D, Barkun A, Bridges R, et al. Canadian Association of Gastroenterology consensus guidelines on safety and quality indicators in endoscopy. Can J Gastroenterol 2012;26:17-31.

7. Malheiro R, de Monteiro-Soares M, Hassan C, Dinis-Ribeiro M. Methodological quality of guidelines in gastroenterology. Endoscopy 2014;46:513-525.

8. Park WG, Shaheen NJ, Cohen J, et al. Quality indicators for EGD. Gastrointest Endosc 2015;81:17-30.

9. Hamashima C, Okamoto M, Shabana M, Osaki Y, Kishimoto T. Sensitivity of endoscopic screening for gastric cancer by the incidence method. Int J Cancer 2013;133:653-659.

10. Choi KS, Suh M. Screening for gastric cancer: the usefulness of endoscopy. Clin Endosc 2014;47:490-496.

11. Park JB, Yang JN, Lim YJ, et al. Survey of endoscope reprocessing in Korea. Clin Endosc 2015;48:39-47.

12. Yeh JM, Ho W, Hur C. Cost-effectiveness of endoscopic surveillance of gastric ulcers to improve survival. Gastrointest Endosc 2010;72:33-43. 